\title{
Characteristics of Elderly Patients with Sarcopenia at Geriatric Outpatient Clinic Dr. Hasan Sadikin General Hospital Period 2012-2014
}

\author{
Sania Putri Darwita, ${ }^{1 *}$ Yuni S. Pratiwi, ${ }^{2}$ Lazuardhi Dwipa ${ }^{3}$ \\ ${ }^{1}$ RSUD Sayang Cianjur, West Java, Indonesia, ${ }^{2}$ Department of Biomedical Sciences Faculty of \\ Medicine Universitas Padjadjaran, Indonesia, ${ }^{3}$ Department of Internal Medicine Faculty of \\ Medicine Universitas Padjadjaran/Dr. Hasan Sadikin General Hospital Bandung, Indonesia
}

\section{Abstract}

Background: Sarcopenia can lead to difficulty in physical activities, therefore, elderly cannot live independently. However, data on sarcopenia are not yet available in Dr. Hasan Sadikin General Hospital. This study was conducted to identify the frequency of occurrence and characteristics of elderly patients with sarcopenia.

Methods: This study used descriptive method and was conducted from May to October 2014. Total sampling method was performed to medical records of elderly patients with sarcopenia at Geriatric Outpatient Clinic Dr. Hasan Sadikin General Hospital during the period 2012-2014. Variables observed were age, gender, body mass index (BMI), activities of daily living (ADL), timed up and go test (TUG), mini nutritional assessment (MNA), disease record, and medication record. Patients with sarcopenia were then grouped based on characteristics of age, gender, BMI, ADL, TUG, MNA, disease record, and medication record.

Results: Out of 255 registered elderly patients, 22(8.63\%) were with sarcopenia and one was excluded due to incomplete data. Sarcopenia was most prevalent in patients aged 80 years and older (15.38\%), and commonly in men (57.1\%). Most patients had hypertension history (66.6\%), multiple comorbidities (61.9\%), normal BMI (33.3\%), normal ADL (85.7\%), normal MNA (76.1\%), and normal TUG scores (80.9\%), and polypharmacy (52.3\%) history.

Conclusions: Sarcopenia is prevalent in men aged 80 years and older. Although sarcopenia patients have hypertension history, multiple comorbidities and history of polypharmacy, most patients live with normal daily activities and have good body mass index, however, quality of life of elderlies needs to be improved further.

Keywords: Elderly, quality of life, sarcopenia

\section{Introduction}

Many changes occur during the aging process, one of them is the gradual loss of skeletal muscle mass which can cause the decrease of muscle strength and physical performance. Muscle strength and physical performance are key components of muscle function. A reduction in skeletal mass and muscle function is known as sarcopenia and is one of the problems faced by the elderly. ${ }^{1}$ Elderlies with sarcopenia have a higher chance of experiencing difficulties or dependency in doing physical activities, thus making the elderly unable to live independently. ${ }^{1}$

There are differences between prevalence of sarcopenia in men and women. Most studies have shown that sarcopenia occurs more often in men than in women even though the number does not differ significantly. In Japan ${ }^{3}$, the prevalence of sarcopenia is $11.3 \%$ and $10.7 \%$, in Taiwan ${ }^{2,4}$ is $9.4 \%$ and $9.8 \%$ for men and women respectively. There is an increase in the occurence of sarcopenia as a person ages. A study done by New Mexico Elder Health Survey reported an increase in the prevalence of sarcopenia with an increase in age, showing percentage of $13-24 \%$ in people of age 70 years or younger and $50 \%$ in people of age 80

Correspondence: Sania Putri Darwita, RSUD Sayang Cianjur, Bojongherang, Kec. Cianjur, Kabupaten Cianjur, West Java 43216, Indonesia, Email: saniaputridarwita@gmail.com *Former Address: Faculty of Medicine Universitas Padjadjaran 
years or older. .-6 $^{-6}$

A study in Rome ${ }^{7}$ reported that the average number of disease in elderlys with sarcopenia and those without sarcopenia are the same, but Parkinson's, Chronic Obstructive Pulmonary Disease, Osteoarthritis, and Cerebrovascular Disease are diseases commonly suffered by patients with sarcopenia. A study in Thailand ${ }^{8}$ showed that sarcopenia is related to higher body mass index (BMI). Meanwhile, a study conducted in Brazil ${ }^{9}$ showed that there is a significant relationship between sarcopenia with the timed up and go test (TUG) score, whereby a lower muscle mass resulted in a higher TUG score. A research in Turkey ${ }^{10}$ reported that elderlys with sarcopenia are mostly dependent and that the mini nutritional assessment (MNA) score is lower in sarcopenia patients who have malnutrition $(10.4 \%)$ and risk of malnutrition (24.6\%). Polypharmacy can cause malnutrition which can lead to sarcopenia. ${ }^{6-11}$

Sarcopenia has become an increasingly important problem, however studies about sarcopenia are rarely performed, especially in Indonesia. Information regarding sarcopenia was not even available at the Geriatric Oupatient Clinic of Dr. Hasan Sadikin General Hospital. The objectives of the study was to identify the frequency of occurence and the characteristics of elderly patients with sarcopenia at the Geriatric Outpatient Clinic of Dr. Hasan Sadikin General Hospital during the period of 2012-2014.

\section{Methods}

This study was conducted using a descriptive method. Total sampling method was performed to the medical records of elderly patients diagnosed with sarcopenia at the Geriatric Outpatient clinic of Dr. Hasan Sadikin General Hospital during the period of 2012-2014. This study was approved by the Health Research Ethics Committee of Dr. Hasan Sadikin General Hospital, Bandung. The rate of occurence and characteristics of elderly patients with sarcopenia was obtained from the medical records of patients with sarcopenia which met the inclusion criteria i.e. the elderly patient's entire medical record with sarcopenia was confirmed with a bioimpedance analysis (BIA) test, a grip strength test and a 6 minutes walking test. Medical records that did not contain data on the age, sex, disease record, BMI, activities of daily living (ADL), MNA, TUG, and medication record were excluded from this study.

From 22 elderly patients with sarcopenia, 21 patients had medical records which met the inclusion criteria. Sarcopenia was defined as the gradual loss of muscle mass as written from the results of a BIA test showing the index value of skeletal muscle less than $7 \mathrm{~kg} / \mathrm{m}^{2}$ for men or less than $5.8 \mathrm{~kg} / \mathrm{m}^{2}$ for women; a loss in the muscle function (strength or performance) showing the results of the grip strength test less than $30.3 \mathrm{~kg}$ for men or less than $19.3 \mathrm{~kg}$ for women; the 6 minutes walking test with a walking rate less than $1.27 \mathrm{~m} / \mathrm{s}$ for men or less than $1.19 \mathrm{~m} / \mathrm{s}$ for women; or are unable to complete this test at all.,12 Sarcopenia was then categorized into (i) presarcopenia of which there was a reduction in the muscle mass, (ii) sarcopenia, the reduction in the muscle mass as well as a loss in the muscle strength or physical performance, and (iii) severe sarcopenia, a loss in muscle mass, muscle strength, and physical performance. ${ }^{13}$

Age was categorized into 'young old' (6069 years old), 'middle old' 70-79 years old) and 'very old' ( $>80$ years old) as described else where. ${ }^{14}$ Disease record was analyzed by looking at the frequency and type of disease that was most common in patients with sarcopenia.

Table 1 Frequency of Occurence of Sarcopenia in Elderly Patients at Geriatric Outpatient Clinic Dr. Hasan Sadikin General Hospital during period 2012-2014 Based on Age Category

\begin{tabular}{|c|c|c|}
\hline \multirow{2}{*}{ Age } & Total respondents & Sarcopenia Frequency \\
\hline & $\mathrm{n}$ & n $(\%)$ \\
\hline 60-69 years old & 109 & $8(7.34)$ \\
\hline 70-79 years old & 133 & $12(9.02)$ \\
\hline$\geq 80$ years old & 13 & $2(15.38)$ \\
\hline Total & 255 & $22(100)$ \\
\hline
\end{tabular}


Body mass index (BMI) was obtained from the ratio of body weight (BW) (in $\mathrm{kg}$ ) with height $(\mathrm{BH})$ squared (in meters). BMI=BW/ $\mathrm{BH}^{2}$, the $\mathrm{BH}$ of elderly people was obtained from the conversion of knee height. BMI was categorized as being underweight $\left(<17 \mathrm{~kg} / \mathrm{m}^{2}\right.$ for women and $<18 \mathrm{~kg} / \mathrm{m}^{2}$ for men), normal (17-23 kg/m $\mathrm{m}^{2}$ for women and $18-25 \mathrm{~kg} / \mathrm{m}^{2}$ for men), overweight ( $>23-27 \mathrm{~kg} / \mathrm{m}^{2}$ for women and $>25-27 \mathrm{~kg} / \mathrm{m}^{2}$ for men), and obese $(>27$ $\mathrm{kg} / \mathrm{m} 2$ for both men and women). ${ }^{15}$

Functional independence in daily personal activities was tested by ADL. The Barthel Index measured eating, bathing, self-care, dressing, urinating, defecating, using the toilet, transfering, mobility, and going up and down the stairs. The ADL was categorized as independent (score 20), mild dependent (12-19), moderate dependent (9-11), severe dependent (5-8) and totally dependent (04). ${ }^{16}$

The nutritional status of elderly people through 18 questions was tested by MNA. The results of the MNA test were categorized as normal nutritional status (score 24-30), risk of malnutrition (17-23.5) and malnutrition (16.5 or less)..$^{17}$

Balance and stability of body posture of the elderly, and specifically assessing the risk of falling was measured by TUG. The subjects

Table 2 Characteristics of Elderly Patients with Sarcopenia at Geriatric Outpatient Clinic Dr. Hasan Sadikin General Hospital during period 2012-2014

\begin{tabular}{|c|c|c|c|}
\hline Variable & $\begin{array}{c}\text { Sarcopenia } \\
(n=5)\end{array}$ & $\begin{array}{c}\text { Severe Sarcopenia } \\
(n=16)\end{array}$ & $\begin{array}{c}\text { Total } \\
(\mathrm{n}=21)\end{array}$ \\
\hline \multicolumn{4}{|l|}{$\operatorname{Sex}(n)$} \\
\hline Male & 2 & 10 & 12 \\
\hline Female & 3 & 6 & 9 \\
\hline \multicolumn{4}{|l|}{ Disease (n) } \\
\hline-2 diseases & 1 & 7 & 8 \\
\hline$>2$ diseases & 4 & 9 & 13 \\
\hline \multicolumn{4}{|l|}{ BMI (n) } \\
\hline Underweight & - & 2 & 2 \\
\hline Normal & 3 & 4 & 7 \\
\hline Overweight & 1 & 5 & 6 \\
\hline Obese & 1 & 5 & 6 \\
\hline \multicolumn{4}{|l|}{ ADL (n) } \\
\hline Independent & 5 & 13 & 18 \\
\hline Mild Dependent & - & 3 & 3 \\
\hline \multicolumn{4}{|l|}{ MNA (n) } \\
\hline Normal & 4 & 11 & 15 \\
\hline Risk of Malnutrition & 1 & 3 & 4 \\
\hline Malnutrition & - & 2 & 2 \\
\hline \multicolumn{4}{|l|}{ TUG (n) } \\
\hline High Risk of Falling & - & 4 & 4 \\
\hline Normal & 5 & 12 & 17 \\
\hline \multicolumn{4}{|l|}{ Medication (n) } \\
\hline Polypharmacy & 3 & 8 & 11 \\
\hline Non-polypharmacy & 2 & 8 & 10 \\
\hline
\end{tabular}


Table 3 Disease Record of Elderly Patients with Sarcopenia

\begin{tabular}{lc}
\hline & Frequency (n) \\
\hline Stroke & 1 \\
Cataract & 5 \\
Angina & 4 \\
Acute Myocardial Infarction & 1 \\
Tuberculosis/Chronic Obstructive Pulmonary Disease/Asthma & 1 \\
Hypercholesterolemia & 6 \\
Hypertriglyceridemia & 2 \\
Obesity and fatness & $12(57.1 \%)^{*}$ \\
Diabetes Melitus & 9 \\
Hypertension & $14(66.6 \%)^{*}$ \\
Urolithiasis & 1 \\
Benign Prostatic Hyperplasia (BPH) & - \\
Liver problems (Urinary Tract Infection, decrease in function) & 1 \\
Osteoporosis & 5 \\
Osteoarthritis & 2 \\
Gout & 1 \\
Anemia & - \\
Cancer & - \\
Gastritis & 1 \\
Hepatitis & 1 \\
Cholelitiasis & 2 \\
TOTAL & 21 \\
\hline
\end{tabular}

Note: * Hypertension and Obesity were the most prevalent diseases in older patients

were asked to stand up from a chair and then asked to walk 3 meters and turn around again until they returned to their initial seat. The results of the TUG test were categorized as having a high risk of falling (score of $>15 \mathrm{~s}$ ) and normal $(<15 \mathrm{~s}){ }^{18}$

The medication record was categorized as polypharmacy (the use of 5 or more medications) and non-polypharmacy. ${ }^{19}$ After the data was collected and categorized, it was presented in frequency table.

\section{Results}

During the period of 2012-2014, 255 patients visited the Geriatric Outpatient Clinic at Dr. Hasan Sadikin General Hospital and $22(8.63 \%)$ of them were diagnosed with sarcopenia, consisting of 13 male $(11.2 \%)$ and 9 female $(6.4 \%)$. However, of the 22 patients with sarcopenia, only 21 patients met the inclusion criteria due to incomplete data.

Based on the age category, sarcopenia occured most often in the age of 80 and above $(15.38 \%)$ as shown in Table 1 . The characteristics of elderly patients with sarcopenia showed that there was no patient found with presarcopenia, and most of the patients had severe sarcopenia, i.e 16 out of $21(76.1 \%)$ patients. Most of the patients were male (12 out of 21 patients), had a history of more than two diseases (12 out of 21 patients), had normal BMI ( 7 out of 21 patients) followed with BMI categorized as overweight and obese (each being 6 out of 21 patients).

The patients with sarcopenia in this study mostly had an ADL value of independent (18 out of 21 patients), had a normal TUG (17 out of 21 patients), had a normal MNA value (16 out of 21 patients), and had a history of polypharmacy (11 out of 21 patients) (Table $2)$. Furthermore, most of the patients $(66.6 \%)$ 
had a history of hypertension (14 out of 21 patients) (Table 3).

\section{Discussion}

The prevalence of sarcopenia in patients attending the Geriatric Outpatient Clinic of Dr. Hasan Sadikin General Hospital during the period of $2012-2014$ is $8.63 \%$, and male is being the most prevalent, in line with study in $\mathrm{Japan}^{3}$ with $11.3 \%$ in males and $10.7 \%$ in females. However, this result differs with a study carried out in Taiwan ${ }^{4}$ that sarcopenia occurs more often in females. Many factors such as hormones, disorder of the nervous system, lack of nutrition, lack of physical activity, or chronic inflammation may play role in sarcopenia. ${ }^{13}$ Interestingly, gender is not reported as a risk factor in the occurence of sarcopenia, thus, the possibility of sarcopenia for both sexes is the same. ${ }^{13}$

Furthermore, sarcopenia in our study occurres more often in patients aged 80 years and older $(15.38 \%)$, that is increasing compared to those occurring in patients aged $60-69$ years $(7.34 \%)$ and aged $70-79$ years $(8.27 \%)$, confirming that the prevalence of sarcopenia occurs as a person ages. This is in line with a study performed by the New Mexico Elder Health Survey which reported an increase in the prevalence of sarcopenia with an increase in age. Between the ages of 50 to 60 years, a decrease in muscle strength occurs as much as $1.5 \%$ per annum and a sharper decrease occurs as much as 3\% per annum for those aged 60 years or older. ${ }^{5-6}$

Patients with sarcopenia at the Geriatric Outpatient clinic mostly had a similar disease record to most patients without sarcopenia, i.e. more than two diseases otherwise known as multiple comorbidity. The average amount of illnesses in elderly patients with sarcopenia and those without sarcopenia are the same. ${ }^{7}$ The patients in this study mostly have a history of hypertension, different then study result in Western countries, showing that Parkinson's, Chronic Obstructive Pulmonary Disease, Osteoarthritis, and Cerebrovascular disease are diseases commonly suffered by patients with sarcopenia. ${ }^{7}$ This might be due to the difference in the population structure and geographic location and food and lifestyle behavior of the population.

Most of the patients with sarcopenia in this study has normal BMI, however, sarcopenia could occur even though patients has normal BMI with a lower muscle mass in body composition and a higher fat content, suggesting an unhealthy lifestyle lacking physical activities. Physical inactivity may cause atrophy or loss in muscle fibers and fat build up, resulting in sarcopenia. ${ }^{6}$ Interestingly, the occurence of sarcopenia in fat and obese patients is also quite high, in line with a study conducted in Thailand ${ }^{8}$, showing that sarcopenia is related with a higher BMI. Obesity is one of the conditions that can trigger the release of IL- 6 which increases glucocortoids and catecolamines, reducing the growth hormones and causing sarcopenia. ${ }^{6,13}$

Elderly people with sarcopenia have a higher risk of suffering from physical disabilities if early intervention is not performed. ${ }^{13}$ There are still debates on the relationship between sarcopenia and functional status. Elderly people with sarcopenia are mostly dependent, but there is no significant relationship between ADL and sarcopenia. ${ }^{10}$ Furthermore, people with sarcopenia do not experience a decrease in functional status. ${ }^{20}$ Based on the ADL value, most of the elderly patients with sarcopenia in this study has an independent functional status. Since the elderly people in this study are still able to come to the outpatient clinic, thus they have a more controlled health and good independence. They may have a high risk of having a decreased ADL in the future, thus, an early intervention should be done to prevent a decrease in independence.

Furthermore, poor nutritional status is one of the factors that can cause sarcopenia and it can result in a decrease of protein synthesis, increase of proteolysis, thus causing a decrease in the muscle mass and function. ${ }^{13}$ Most of the patients with sarcopenia in this study has a normal nutritional status. The results of this study differs with the results of a study conducted in Turkey ${ }^{10}$ that showed a low MNA score in sarcopenia patients who has malnutrition and has risk of malnutrition. This difference may have been because the study conducted in Turkey ${ }^{10}$ is a community based population, instead of out-patients in our study, who has more controlled health and thus probably had better nutritional status. Poor nutritional status is just one of the risk factors of sarcopenia; in this study poor nutrition is probably not the main factor causing sarcopenia thus further research is needed on other factors which may have a more significant role in causing sarcopenia.

The reduction in muscle mass and function can cause an increase in the risk of falling in elderly people. There is a significant relationship between sarcopenia with the TUG score, resulting in a lower muscle mass results 
in a higher TUG score. ${ }^{9}$ In this study, most of the patients still had a normal TUG score. This differs with the result of the study in Brazil ${ }^{9}$ which is carried out in the community where the elderly people with sarcopenia already has a higher risk of falling.

Most of the patients with sarcopenia in this study has a history of polypharmacy, that may lead to malnutrition problems. Elderlies with polypharmacy may have a decrease in fiber, vitamin, and mineral intakes as well as an increase in the consumption of cholesterol, glucose, and sodium. An increase in the consumption of saturated fats, carbohydrates and cholesterols along with a decrease in fibers and proteins can result in a deterioration of the physical health. ${ }^{11}$ Malnutrition or the lack of vitamin D consumption itself can be one of the causes of sarcopenia. ${ }^{6}$

Pre-sarcopenia is not prevalent in our study, however, most of the patients suffer from severe sarcopenia. A majority of the patients has more than two diseases, similar to most of the other patients at the Geriatric Outpatient clinic without sarcopenia, with the most prevalent disease such as hypertension and polypharmacy.

The limitations of this study, among others, that the medical history of older patients are not complete. The data base of medical history need to be enhances since the cohort study for older people is interesting to be explored.

In this study, the patients with sarcopenia were described as elderly patients who looked normal, had normal BMI, normal TUG scores, normal nutritional status, and were still able to carry out their daily activities well. This should increase the amount of awareness and care in detecting sarcopenia at an earlier stage in elderlys who still look healthy and independent so that these elderly people can uphold their independence for as long as possible.

In conclusion, sarcopenia is prevalent in male aged 80 years and older. Although sarcopenia patients have history of hypertension, polypharmacy, and multiple comorbidities, most patients live with normal daily activities and have a good body mass index, however, the quality of life of the elderly needs to be improved further. In addition, further study should be conducted regarding the occurrence of sarcopenia in communities, since other studies have shown that the frequency of incidence of sarcopenia is higher in communities. It would be better if the study could be conducted in West Java or integrated across Indonesia. From such a study, it is hoped that we can know the prevalence of sarcopenia in a much larger range, i.e. West Java or Indonesia, and thus prevention and intervention of sarcopenia can be performed so that the elderly have a better quality of life.

\section{References}

1. Chien MY, Kuo HK, Wu YT. Sarcopenia, cardiopulmonary fitness, and physical disability in community-dwelling elderly people. Phys Ther. 2010;90(9):1277-87

2. Lau EMC, Lynn HSH, Woo JW, Kwok TCY, Melton LJ, III. Prevalence of and risk factors for sarcopenia in elderly Chinese men and women. J Gerontol A Biol Sci Med Sci 2005;60A(2):213-6.

3. Tanimoto Y, Watanabe M, Sun W, Sugiura Y, Tsuda Y, Kimura M, et al. Association between sarcopenia and higher-level functional capacity in daily living in community-dwelling elderly subjects in Japan. Arch Gerontology Geriatr. 2012;55(2):e9-e13.

4. Liu LK, Lee WJ, Chen LY, Hwang AC, Lin MH, Peng LN, et al. Sarcopenia, and its association with cardiometabolic and functional characteristics in Taiwan: results from I-Lan Longitudinal Aging Study. Geriatr Gerontol Int. 2014;14(S1):36-45.

5. von Haehling S, Morley JE, Anker SD. An overview of sarcopenia: facts and numbers on prevalence and clinical impact. J Cachexia Sarcopenic Muscle. 2010;1(2):129-33

6. Baumgartner RN, Waters DL. Sarcopenia and sarcopenic-obesity. In: Pathy MSJ, Sinclair AJ, Morley JE, editors. Principles and Practice of Geriatric Medicine. 4th ed. Chichester,West Sussex,England: John Wiley \& Sons Ltd; 2006. p. 909-47.

7. Landi F, Liperoti R, Fusco D, Mastropaolo S, Quattrociocchi D, Proia A, et al. Prevalence and risk factors of sarcopenia among nursing home older residents. J Gerontol A Biol Sci Med Sci. 2012;67(1):48-55.

8. Pongchaiyakul C, Limpawattana P, Kotruchin P, Rajatanavin R. Prevalence of sarcopenia and associated factors among Thai population. J Bone Miner Metab. 2013;31(3):346-50.

9. Falsarella GR, Coimbra IB, Barcelos CC, Iartelli I, Montedori KT, Santos MNJ, et al. Influence of muscle mass and bone mass on the mobility of elderly women: an observational study. BMC Geriatr. 2014;14:13.

10. Bahat G, Saka B, Tufan F, Akin S, Sivrikaya 
S, Yucel N, et al. Prevalence of sarcopenia and its association with functional and nutritional status among male residents in a nursing home in Turkey. Aging Male. 2010;13(3):211-4.

11. Heuberger RA, Caudell K. Polypharmacy and nutritional status in elderlys. Drugs Aging. 2011;28(4):315-23.

12. Chen LK, Liu LK, Woo J, Assantachai P, Auyeung TW, Bahyah KS, et al. Sarcopenia in Asia: consensus report of the Asian Working Group for Sarcopenia. J Am Med Dir Assoc. 2014;15(2):95-101.

13. Cruz-Jentoft AJ, Baeyens JP, Bauer JM, Boirie Y, Cederholm T, Landi F, et al. Sarcopenia: European consensus on definition and diagnosis: report of the European Working Group on Sarcopenia in Older People. Age Ageing. 2010;39(4):412-23.

14. Stuart-Hamilton I. The psychology of ageing: An Introduction. $5^{\text {th }}$ ed. London: Jessica Kingsley Publishers; 2012.

15. Kementrian Kesehatan Republik Indonesia. Indeks massa tubuh. 2014 [cited 2014 February 18]. Available from: http://www. depkes.go.id/index.php?vw=2\&id=A-137.

16. Houlden $\mathrm{H}$, Edwards $\mathrm{M}$, McNeil J, Greenwood R. Use of the barthel index and the functional independence Measure during early inpatient rehabilitation after single incident brain injury. Clin Rehab. 2006;20(2):153-9.

17. Tsai AC, Hsu HY, Chang TL. The Mini Nutritional Assessment (MNA) is useful for assessing the risk of malnutrition in adults with intellectual disabilities. J Clin Nurs. 2011;20(23-24):3295-303.

18. Reuben DB, Rosen S. Principles of Geriatric Assessment. In: Halter JB, Ouslander JG, Tinetti ME, Studenski S, High KP, Asthana $\mathrm{S}$, editors. Hazzard's geriatric medicine and gerontology. $6^{\text {th }}$ ed. United States: The McGraw-Hill Companies, Inc; 2009. p. 141-5.

19. Viktil KK, Blix HS, Moger TA, Reikvam A. Polypharmacy as commonly defined is an indicator of limited value in the assessment of drug-related problems. Br J Clin Pharmacol. 2007;63(2):187-95.

20. Rolland Y, Lauwers-Cances V, Cristini C, van Kan GA, Janssen I, Morley JE, et al. Difficulties with physical function associated with obesity, sarcopenia, and sarcopenic-obesity in communitydwelling elderly women: the EPIDOS (EPIDemiologie de l'OSteoporose) Study. Am J Clin Nutr. 2009;89(6):1895-900. 